\title{
Clinical characteristics and genetic analyses in a Chinese family affected by primary aldosteronism: a case report
}

\author{
Jieyi $\mathrm{Li}^{1,2}$, Junfang Zhao ${ }^{3}$, Wentao $\mathrm{He}^{1,2 \#}$, Gang Yuan ${ }^{1,2 \#}$ \\ ${ }^{1}$ Department of Endocrinology, Tongji Hospital, Tongji Medical College, Huazhong University of Science and Technology, Wuhan, China; ${ }^{2}$ Branch \\ of National Clinical Research Center for Metabolic Disease, Wuhan, China; ${ }^{3}$ Department of Biliary and Pancreatic Surgery, Tongji Hospital, Tongji \\ Medical College, Huazhong University of Science and Technology, Wuhan, China \\ \#These authors contributed equally to this work. \\ Correspondence to: Dr. Wentao He. Department of Endocrinology, Tongji Hospital, Tongji Medical College, Huazhong University of Science and \\ Technology. No.1095 Jiefang Road, Wuhan, China. Email: wthe@tjh.tjmu.edu.cn.
}

\begin{abstract}
The clinical and aetiological characteristics of family-clustered primary aldosteronism (PA) are not fully understood and need further exploration. Our study reported a PA case with a family history accompanied by unusual concomitant disease and explored the genetic background of the affected family members, thus providing more evidence of the manifestation and pathogenesis of family-clustered PA. We studied a family with PA in which the proband and her maternal aunt were diagnosed with aldosteroneproducing adenoma (APA) and primary adrenal hyperplasia (PAH), respectively. Apart from the diagnosis of APA, the proband also had a history of craniopharyngioma. Both patients achieved desirable blood pressure control and potassium levels after laparoscopic unilateral adrenalectomy. Multiple-gene panel analysis was applied in both resected adrenal lesions and peripheral blood of the proband to screen potential genetic variants. Then, the detected variant was verified by Sanger sequencing in her maternal aunt. No phenotyperelated germline mutation was detected in the two affected patients. One somatic nonsense mutation (L168R) of KCNJ 5 was detected in the DNA of resected APA from the proband, whereas her maternal aunt did not carry the same somatic mutation. Although no identical mutation was found in the two patients, it remains unknown whether certain unmeasured genetic or epigenetic factors are involved in the development of family-clustered PA. Further studies focused on PA cases with complex manifestations or with a family history will be needed to expand our knowledge of the pathogenesis of PA.
\end{abstract}

Keywords: Primary aldosteronism (PA); family history; craniopharyngioma; genetic analyses; case report

Submitted Dec 01, 2020. Accepted for publication Feb 03, 2021.

doi: 10.21037/apm-20-2392

View this article at: http://dx.doi.org/10.21037/apm-20-2392

\section{Introduction}

Primary aldosteronism (PA) is the most common cause of secondary hypertension and has been strongly linked to an increased risk of cardiovascular events, diabetes mellitus and metabolic syndrome (1). PA is characterized by aberrant secretion of aldosterone, irrespective of low renin and angiotensin levels. Its typical clinical features include resistant hypertension and hypokalemia. While most PA cases are sporadic, approximately $5 \%$ of all PA patients show familial inheritance, which is referred to as familial hyperaldosteronism (FH) (2). FH type I (FH-I), also called glucocorticoid-remediable aldosteronism (GRA), is caused by an unequal cross-over of the CYP11B1 gene (encoding steroid $11 \beta$-hydroxylase) and CYP11B2 gene (encoding aldosterone synthase) (3). This unequal cross-over leads to ectopic synthesis of aldosterone through the regulation of adrenocorticotropic hormone (ACTH), which could be inhibited by glucocorticoid management. FH type III (FH-III) has been shown to be associated with mutations in the KCN75 gene (encoding an inwardly rectifying potassium channel) (4), which causes sodium influx and 

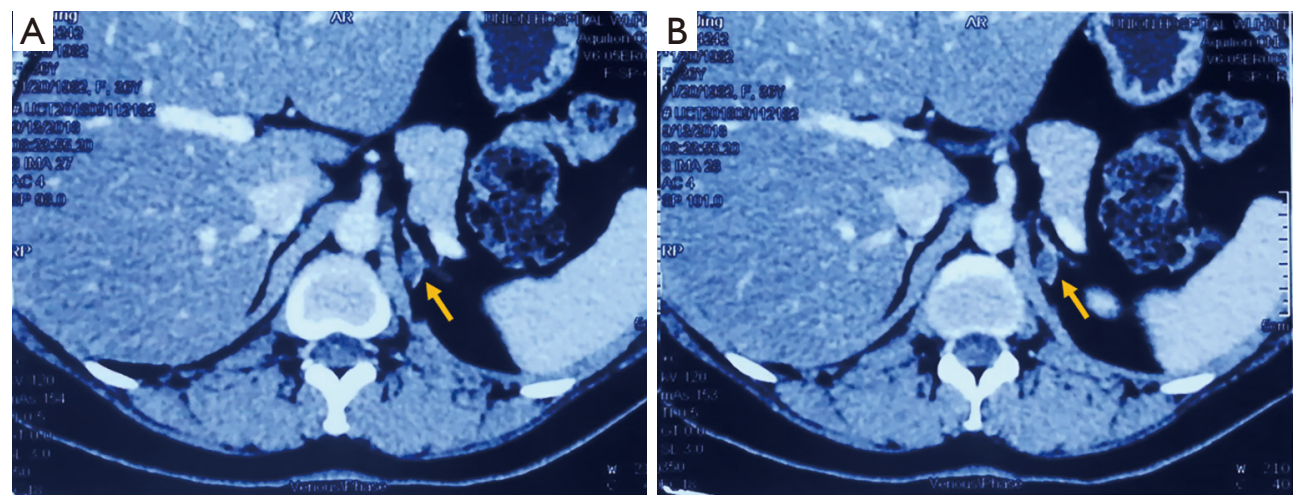

Figure 1 The abdominal computed tomography (CT) scan of the proband patient. CT showing adenoma (max cross-section $1.4 \mathrm{~cm} \times 0.6 \mathrm{~cm}$; yellow arrow) in left adrenal gland.

cell depolarization, resulting in constitutive aldosterone production. The phenotype of FH-III shows variability due to different KCNJ5 mutations. A severe type of FH-III usually has childhood onset, displays severe hypertension and hypokalaemia, and is resistant to antihypertensives, including aldosterone antagonists, while some cases of FH-III exhibit a mild phenotype and even lack radiologic findings in adrenals. Some familial PAs are linked to mutations in other ion channel genes, such as CACNA1H (encoding T-type calcium channel subunit Cav3.2) and CACNA1D (encoding L-type calcium channel subunit Cav1.3) $(5,6)$. There is another group of inherited PA, which has been referred to as $\mathrm{FH}$ type II (FH-II). Unlike FH-I, they are nonglucocorticoidremediable, and negative for GRA gene mutation testing. FH-II families may display as aldosterone-producing adenoma (APA) and adrenal hyperplasia, which is clinically indistinguishable from sporadic PAs. The molecular basis of FH-II has been studied for decades. It has been reported that chromosomal region 7p22 is linked to FH-II (7). More recently, a germline mutation in the CLCN2 gene, which encodes the inwardly rectifying chloride channel CLC2, was identified in some FH-II cases (8). However, there still remains a group of inherited cases without the CLCN2 mutation and that cannot be explained by known genetic causes. Therefore, the aetiology of familial PAs still needs further exploration.

Herein we reported the genetic analyses in two related PA patients using multiple-gene panel analyses, aiming to provide more data on PA cases with a family history.

We present the following case in accordance with the CARE reporting checklist (available at http://dx.doi. org/10.21037/apm-20-2392).

\section{Case presentation}

A 36-year-old female was referred to our department on 8 Oct. 2018 for an incidentaloma within the left adrenal gland. She had undergone open surgery for craniopharyngioma five years before admission. After the operation, she started to take prednisone ( $5 \mathrm{mg} /$ day), L-thyroxine $(50 \mu \mathrm{g} /$ day $)$ and desmopressin acetate $(200 \mu \mathrm{g} /$ day $)$ as replacement regimens. Hypertension was found two years later during her regular follow-up. She began to suffer from intermittent lower extremity weakness, which was caused by hypokalaemia (with her lowest serum potassium level at $2.83 \mathrm{mmol} / \mathrm{L}$ ). Computed tomography (CT) showed a low-density nodule (maximum cross-section $1.4 \mathrm{~cm} \times 0.6 \mathrm{~cm}$ ) in the medial extremity of the left adrenal gland (Figure 1). Her mother had hypertension and died for unknown reasons. Her maternal aunt (the sister of her mother) was diagnosed with PA at the age of 64 (Figure 2).

The blood pressure (BP) of the proband was controlled at $121-138 / 86-102 \mathrm{mmHg}$ with nifedipine $(30 \mathrm{mg} /$ day) Serum potassium was $2.84 \mathrm{mmol} / \mathrm{L}$ on admission, with urinary potassium excretion at $62.65 \mathrm{mmol} / 24 \mathrm{~h}$. She had a high level of plasma aldosterone, with an aldosterone-renin ratio (ARR) of $56.8 \mathrm{pg} / \mu \mathrm{IU}$ (Table 1), and postoperative anterior pituitary hypofunction was observed (Table 1). Catecholamines were within the normal range (Table 1). The saline infusion test (SIT) showed unsuppressed aldosterone levels (Table 2). Moreover, a low-dose dexamethasone inhibition test was performed to preliminarily exclude GRA. No suppression of aldosterone levels was observed after 2 days of dexamethasone administration $(0.5 \mathrm{mg} / 6 \mathrm{~h})$ (Table 2).

Selective adrenal venous sampling (AVS) indicated 


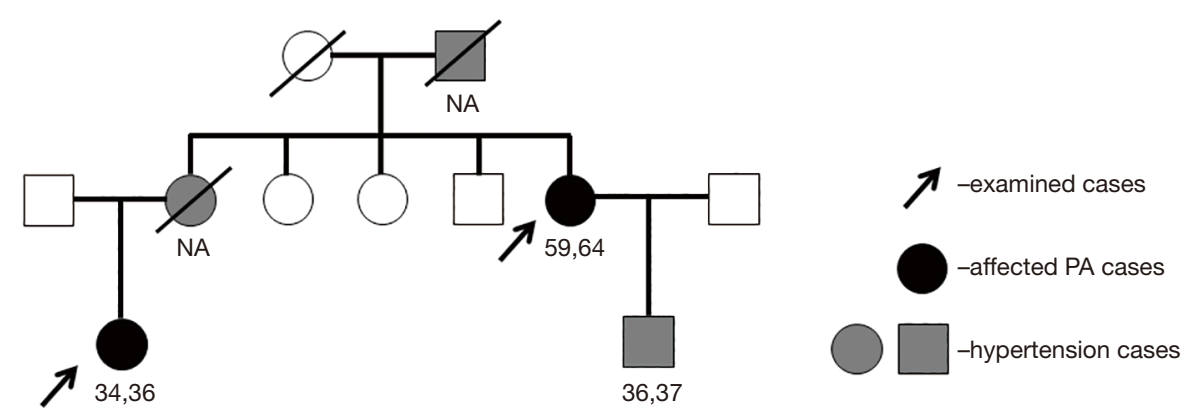

Figure 2 Pedigree chart. Numbers below the case indicate the onset age and current age (year), respectively. NA, not available.

Table 1 Hormone profiles of the proband

\begin{tabular}{lcc}
\hline Parameter & Value & Reference \\
\hline Aldosterone $(\mathrm{pg} / \mathrm{mL})$ & 318 & $0-353.0$ \\
Renin activity $(\mu \mathrm{lU} / \mathrm{mL})$ & 5.6 & $4.4-46.1$ \\
ARR & 56.8 & $<30$ \\
ACTH $(\mathrm{pg} / \mathrm{mL})$ & 20.9 & $7.2-63.3$ \\
Urinary metanephrine $(\mu \mathrm{g} / 24 \mathrm{~h})$ & 51.48 & $38-266$ \\
Urinary normetanephrine $(\mu \mathrm{g} / 24 \mathrm{~h})$ & $2,427.11$ & $27-561$ \\
\hline
\end{tabular}

ARR, aldosterone-renin ratio; $\mathrm{ACTH}$, adrenocorticotropic hormone.

lateralized secretion of aldosterone from the left adrenal gland, with a cortisol-corrected aldosterone ratio of 72 relative to the right. A laparoscopic left adrenalectomy was subsequently performed. Macroscopic analysis of the resected adrenal revealed one single yellow nodule $(12 \mathrm{~mm} \times 6 \mathrm{~mm} \times 5 \mathrm{~mm})$, and microscopic analysis showed zona-fasciculata-like cells, which are part of the diagnosis of APA (Figure 3). After surgery, the serum aldosterone and potassium levels were restored to normal ranges (aldosterone at $21 \mathrm{pg} / \mathrm{mL}$ and potassium at $4.13 \mathrm{mmol} / \mathrm{L}$ ), and blood pressure was well maintained without antihypertensives. The patient also reported relief of lower limb fatigue and improvement in quality of life.

The proband's aunt was diagnosed with hypertension at the age of 59. Despite the combined use of calcium channel blockers, angiotensin receptor blockers and diuretics, her blood pressure remained high $(150-160 / 80-90 \mathrm{mmHg})$. At the age of 64 , she was investigated for possible secondary hypertension. Abdominal CT revealed nodular hyperplasia in the left adrenal gland (Figure 4A), and the PA screening test showed a plasma aldosterone level of $479 \mathrm{pg} / \mathrm{mL}$ (normal range $<353.0 \mathrm{pg} / \mathrm{mL}$ ) with an ARR ratio of $41.24 \mathrm{pg} / \mu \mathrm{IU}$. Although the patient claimed no symptoms of weakness,
Table 2 Endocrine function tests of the proband

\begin{tabular}{lcc}
\hline Test & Before & After \\
\hline Dexamethasone suppression test & & \\
Cortisol $(\mu \mathrm{g} / \mathrm{L})$ & 44 & 9 \\
Aldosterone $(\mathrm{pg} / \mathrm{mL})$ & 307 & 326 \\
Saline infusion test $(\mathrm{SIT})$ & & \\
Cortisol $(\mu \mathrm{g} / \mathrm{L})$ & 3.44 & 3.84 \\
Aldosterone $(\mathrm{pg} / \mathrm{mL})$ & 180 & 289 \\
\hline
\end{tabular}

biochemical tests showed a low serum potassium level of $3.16 \mathrm{mmol} / \mathrm{L}$. Laparoscopic adrenalectomy revealed extensive nodular hyperplasia in the left adrenal gland (Figure 4B). Satisfactory blood pressure was achieved by a single antihypertensive after surgery.

Concerning family aggregation, we further explored the genetic profiles in these two patients. A panel of 5,000 genes involved in endocrine and metabolic diseases was analysed by exon-capture high-throughput sequencing (EC-HTS) using the Illumina HiSeq platform (https://cdn.amegroups. cn/static/public/apm-20-2392-1.xlsx). The results revealed a somatic KCN75 mutation c.503T>G (p. Leu168Arg) in the resected nodule (Figure 5). No germline mutations related to PA were detected. Sanger sequencing of DNA samples from both blood and resected adrenal lesions was applied to her aunt for verification, and no suspected mutation was detected.

All procedures performed in studies involving human participants were in accordance with the ethical standards of the ethics committee of Tongji Hospital, Tongji Medical College, Huazhong University of Science and Technology (IRB ID: TJ-C20210103) and with the Helsinki Declaration (as revised in 2013). Written informed consent was obtained from the patient. 


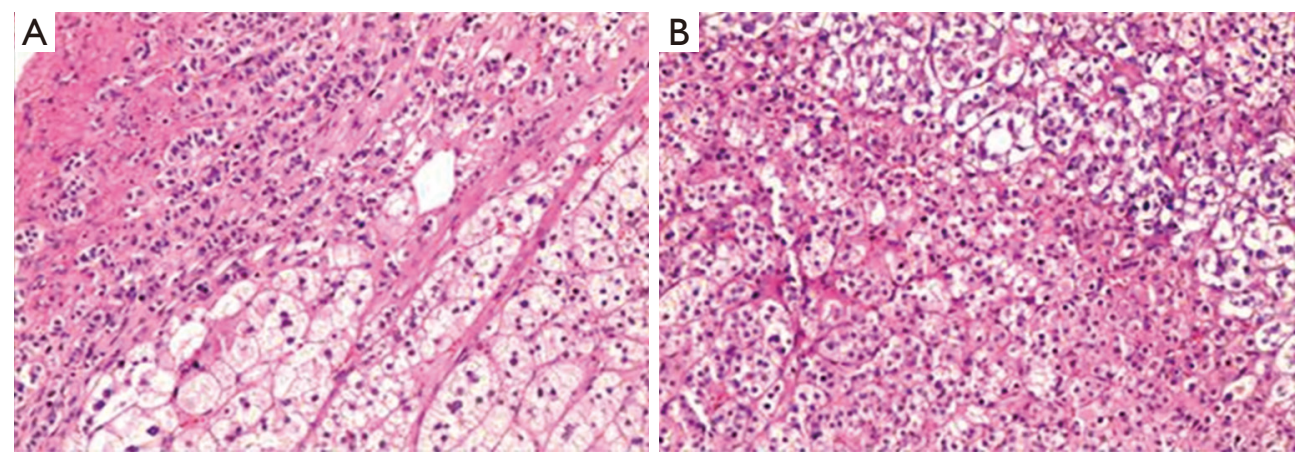

Figure 3 The photograph of surgical specimen of the proband patient. Hematoxylin and eosin (H\&E) stain of paraffin embedded adenoma tissue $(\times 100)$.
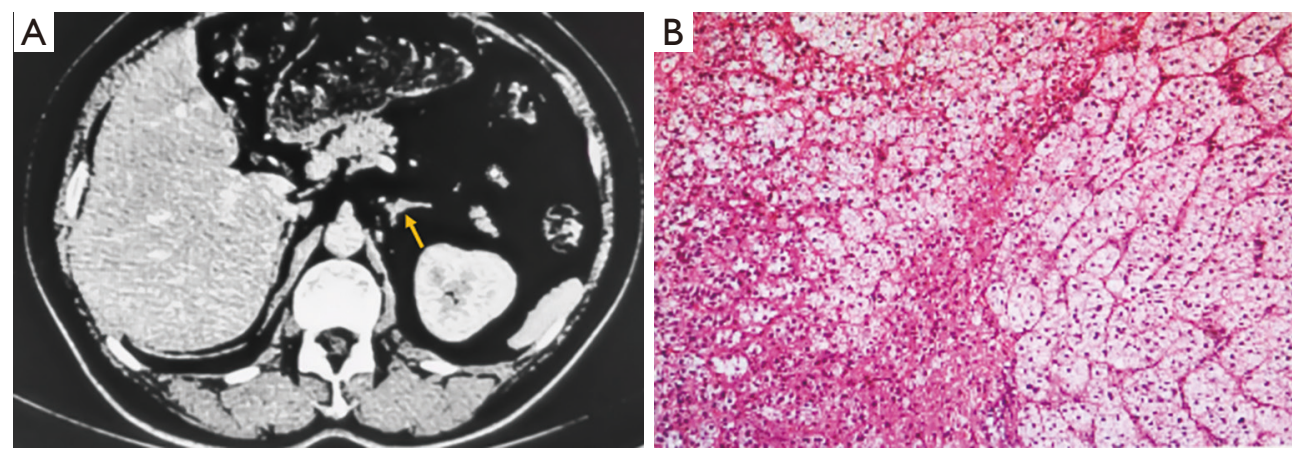

Figure 4 The abdominal computed tomography (CT) scan and photograph of surgical specimen of the maternal aunt of the patient. (A) CT showing nodular hyperplasia in the left adrenal gland (yellow arrow). (B) Hematoxylin and eosin (H\&E) stain of paraffin embedded resected adrenal tissue showing nodular hyperplasia of the adrenal cortex $(\times 100)$.

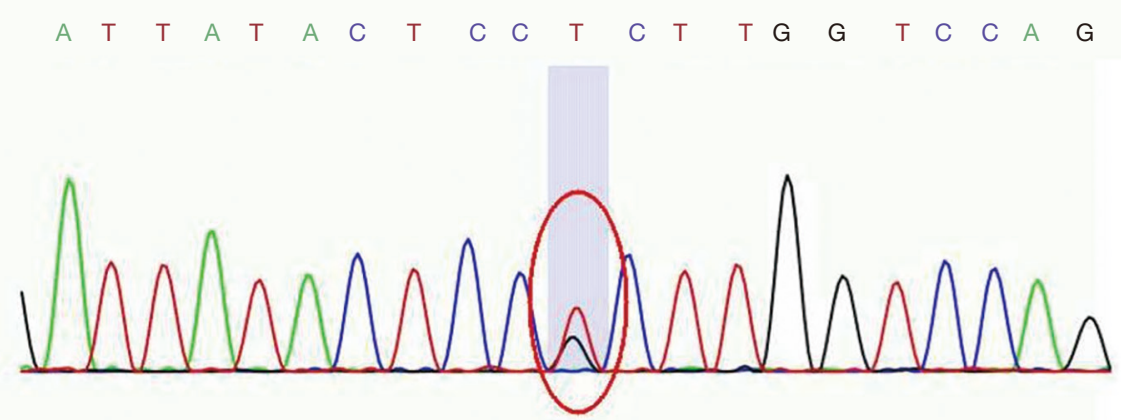

Figure 5 Gene analysis on the resected adenoma tissue of the proband. Gene analysis of KCNF5 revealed a missense mutation from CTC to CGC at codon 168 , leading to a substitution of leucine to arginine.

\section{Discussion}

Currently, the genetic causes of a great number of familial PA cases are still unknown. In our study, we investigated the genetic alterations in a family affected by PA. Although no germline mutation was found in this family, a somatic KCNJ5 mutation (L168R) was detected in one of the affected cases with APA.

Regarding FH, it is expected that the affected family 
members share the same germline mutation. However, no pathogenic germline mutation was detected in our cases, and thus it could not explain the hereditary pattern in this family. Our observation was in line with the study by Mulatero et al. (9), which investigated KCNJ5 mutations in several European families with nonglucocorticoid remediable form of FH. They observed only somatic mutations, instead of germline mutations, in certain patients other than their affected family members. One possible explanation is that those patients in one family were occasionally sporadic forms of PA. For a long time, the diagnosis of FH-II was made based on two or more affected family members suffering PA without identified pathogenic gene mutations. Currently, as more accessible and efficient screenings are being performed, the incidence of PA has been largely underestimated (10). In our report, there is no sufficient evidence for these two cases being familial. Thus, they could possibly be sporadic PAs. Nevertheless, genetic factors cannot fully explain the inheritance of PA. Previous studies have shown aberrant DNA methylation status in adrenocortical adenoma and APA (11-14), indicating epigenetic factors could be taken into consideration during the etiological investigations.

In our report, the patient with APA showed a complex clinical presentation, as she had a prior diagnosis of craniopharyngioma and followed postoperative hypopituitarism. Currently, no evident connection has been made between craniopharyngioma and PA, and further evidence will be needed to explore the possible link between these two diseases. Moreover, hypopituitarism may worth consideration in the reported case, as several pituitary hormones have been shown to be involved in the coordination of aldosterone secretion. Notably, ACTH is one of the physiological regulators of aldosterone production in addition to potassium and angiotensin II. Several studies have demonstrated endogenous ACTH as an important aldosterone secretagogue in PA (15), and a low-dose dexamethasone application $(0.75-2.0 \mathrm{mg} / \mathrm{d}$ for 2 days $)$ could decrease aldosterone levels by a mean of $49 \%$ in a group of 15 patients with APA (16). In addition, vasopressin and TSH both have stimulating effects on aldosterone secretion $(17,18)$. In the current APA patient, low pituitary hormones, including ACTH, TSH and vasopressin, were expected and reflected by symptom manifestation and laboratory tests. Despite the usage of a low-dose vasopressin analogue, it is speculated that this physiological dosage might not significantly affect aldosterone secretion, especially its reaction to SIT. Therefore, the PA diagnosis seems not to be affected by hypopituitarism. However, whether these hormone changes trigger subsequent pathologic changes and drive APA formation still needs further exploration.

Our study suggests that further studies focusing on PA cases with unusual presentations warrant further exploration.

\section{Acknowledgments}

The wordings of this manuscript were edited by the Am J Expert medical editing service.

Funding: This work was supported by the Natural Science Foundation of China [grant number. 81770817]. The funders had no role on the study design, data analysis, interpretation and writing the manuscript in this study.

\section{Footnote}

Reporting Checklist: The authors have completed the CARE reporting checklist. Available at http://dx.doi.org/10.21037/ apm-20-2392

Peer Review File: Available at http://dx.doi.org/10.21037/ apm-20-2392

Conflicts of Interest: All authors have completed the ICMJE uniform disclosure form (available at http://dx.doi. org/10.21037/apm-20-2392). The authors have no conflicts of interest to declare.

Ethical Statement: The authors are accountable for all aspects of the work in ensuring that questions related to the accuracy or integrity of any part of the work are appropriately investigated and resolved. All procedures performed in studies involving human participants were in accordance with the ethical standards of the ethics committee of Tongji Hospital, Tongji Medical College, Huazhong University of Science and Technology (IRB ID: TJ-C20210103) and with the Helsinki Declaration (as revised in 2013). Written informed consent was obtained from the patient.

Open Access Statement: This is an Open Access article distributed in accordance with the Creative Commons Attribution-NonCommercial-NoDerivs 4.0 International License (CC BY-NC-ND 4.0), which permits the noncommercial replication and distribution of the article with the strict proviso that no changes or edits are made and the 
original work is properly cited (including links to both the formal publication through the relevant DOI and the license). See: https://creativecommons.org/licenses/by-nc-nd/4.0/.

\section{References}

1. Monticone S, D'Ascenzo F, Moretti C, et al. Cardiovascular events and target organ damage in primary aldosteronism compared with essential hypertension: a systematic review and meta-analysis. Lancet Diabetes Endocrinol 2018;6:41-50.

2. Mulatero P, Tizzani D, Viola A, et al. Prevalence and characteristics of familial hyperaldosteronism: the PATOGEN study (Primary Aldosteronism in TOrinoGENetic forms). Hypertension 2011;58:797-803.

3. Lifton RP, Dluhy RG, Powers M, et al. A chimaeric 11 beta-hydroxylase/aldosterone synthase gene causes glucocorticoid-remediable aldosteronism and human hypertension. Nature 1992;355:262-5.

4. Choi M, Scholl UI, Yue P, et al. K+ channel mutations in adrenal aldosterone-producing adenomas and hereditary hypertension. Science 2011;331:768-72.

5. Scholl UI, Stolting G, Nelson-Williams C, et al. Recurrent gain of function mutation in calcium channel CACNA1H causes early-onset hypertension with primary aldosteronism. Elife 2015;4:e06315.

6. Scholl UI, Goh G, Stolting G, et al. Somatic and germline CACNA1D calcium channel mutations in aldosteroneproducing adenomas and primary aldosteronism. Nat Genet 2013;45:1050-4.

7. So A, Duffy DL, Gordon RD, et al. Familial hyperaldosteronism type II is linked to the chromosome 7 p22 region but also shows predicted heterogeneity. J Hypertens 2005;23:1477-84.

8. Scholl UI, Stolting G, Schewe J, et al. CLCN2 chloride channel mutations in familial hyperaldosteronism type II. Nat Genet 2018;50:349-54.

9. Mulatero P, Tauber P, Zennaro MC, et al. KCNJ5 mutations in European families with nonglucocorticoid

Cite this article as: Li J, Zhao J, He W, Yuan G. Clinical characteristics and genetic analyses in a Chinese family affected by primary aldosteronism: a case report. Ann Palliat Med 2021;10(7):8378-8383. doi: 10.21037/apm-20-2392 remediable familial hyperaldosteronism. Hypertension 2012;59:235-40.

10. Hannemann A, Wallaschofski H. Prevalence of primary aldosteronism in patient's cohorts and in population-based studies--a review of the current literature. Horm Metab Res 2012;44:157-62.

11. Rechache NS, Wang Y, Stevenson HS, et al. DNA methylation profiling identifies global methylation differences and markers of adrenocortical tumors. J Clin Endocrinol Metab 2012;97:E1004-13.

12. Fonseca AL, Kugelberg J, Starker LF, et al. Comprehensive DNA methylation analysis of benign and malignant adrenocortical tumors. Genes Chromosomes Cancer 2012;51:949-60.

13. Howard B, Wang Y, Xekouki P, et al. Integrated analysis of genome-wide methylation and gene expression shows epigenetic regulation of CYP11B2 in aldosteronomas. J Clin Endocrinol Metab 2014;99:E536-43.

14. Murakami M, Yoshimoto T, Nakabayashi K, et al. Integration of transcriptome and methylome analysis of aldosterone-producing adenomas. Eur J Endocrinol 2015;173:185-95.

15. Sonoyama T, Sone M, Tamura N, et al. Role of endogenous ACTH on circadian aldosterone rhythm in patients with primary aldosteronism. Endocr Connect 2014;3:173-9.

16. Litchfield WR, New MI, Coolidge C, et al. Evaluation of the dexamethasone suppression test for the diagnosis of glucocorticoid-remediable aldosteronism. J Clin Endocrinol Metab 1997;82:3570-3.

17. Nicolini G, Balzan S, Morelli L, et al. LH, progesterone, and TSH can stimulate aldosterone in vitro: a study on normal adrenal cortex and aldosterone producing adenoma. Horm Metab Res

2014;46:318-21.

18. Perraudin V, Delarue C, Lefebvre H, et al. Evidence for a role of vasopressin in the control of aldosterone secretion in primary aldosteronism: in vitro and in vivo studies. $\mathrm{J}$ Clin Endocrinol Metab 2006;91:1566-72. 